\title{
DESENVOLVIMENTO E VALIDAÇÃO DE UM MÉTODO ANALÍTICO PARA A DETERMINAÇÃo DE HISTAMINA EM VINHOS UTILIZANDO CROMATOGRAFIA LÍQUIDA DE ALTA EFICIÊNCIA COM DETECÇÃO POR FLUORESCÊNCIA
}

\author{
Osmar D. Prestes, Michele A. Presta, Diana I. S. Kolberg e Renato Zanella* \\ Departamento de Química, Universidade Federal de Santa Maria, 97105-900 Santa Maria - RS, Brasil \\ Simone B. Rossato, Neidi G. Penna e Luisa H. R. Hecktheuer \\ Departamento de Tecnologia e Ciência dos Alimentos, Universidade Federal de Santa Maria, 97105-900 Santa Maria - RS, Brasil
}

Recebido em 15/7/05; aceito em 2/5/06; publicado na web em 26/9/06

\begin{abstract}
DEVELOPMENT AND VALIDATION OF AN ANALYTICAL METHOD FOR THE DETERMINATION OF HISTAMINE IN WINES USING HIGH PERFORMANCE LIQUID CHROMATOGRAPHY WITH FLUORESCENCE DETECTION. A rapid and efficient method for the analysis of histamine in wines using HPLC with fluorescence detection after derivatization was developed and validated. The method LOD and LOQ values were 0.25 and $0.50 \mathrm{mg} \mathrm{L}^{-1}$ respectively. The repeatability and intermediary precision for the instrument and for the method presented RSD values of 3.7 and $2.9 \%$, and 6.0 and $5.6 \%$, respectively. The recoveries were 95.5 and $89.9 \%$ for the fortification levels of 2 and $10 \mathrm{mg} \mathrm{L}^{-1}$. The method was applied to determine the histamine content in Cabernet Sauvignon wines, which presented values between 1.2 and $5.7 \mathrm{mg} \mathrm{L}^{-1}$.
\end{abstract}

Keywords: histamine; wine; HPLC-fluorescence.

\section{INTRODUÇÃO}

Nos últimos anos, as novas tendências em segurança alimentar e a exigência do consumidor por alimentos saudáveis e de qualidade estão promovendo um aumento na pesquisa por constituintes presentes em alimentos e bebidas que podem afetar a saúde humana. Assim, a presença de substâncias que são fisiologicamente ativas no metabolismo humano tem sido extensivamente estudada em alimentos. Em vinhos, interesse especial tem sido dedicado às aminas bioativas ou biogênicas e, em particular, à histamina ${ }^{1}$. Embora estas substâncias estejam presentes no vinho geralmente em baixas quantidades, elas exibem interação com o metabolismo humano, fato que justifica a pesquisa. A histamina produz dilatação dos vasos sangüíneos e, conseqüentemente, cefaléia. A eliminação ou a redução desta substância nos vinhos tem sido uma prática cada vez mais utilizada em certos países²

A presença de histamina em vinhos está sendo relacionada às más condições higiênico-sanitárias no processo de produção, uma vez que esta substância é formada por algumas espécies de microorganismos. Devido a isso, é importante uma verificação do conteúdo de histamina em vinhos como forma de detectar possíveis falhas no processo de produção ${ }^{3}$. Entretanto, a quantificação de rotina destas substâncias não é utilizada no controle de qualidade dos vinhos, principalmente devido às dificuldades analíticas, como presença de interferentes e complexidade da matriz ${ }^{4}$.

As técnicas de separação de aminas empregadas para a determinação de histamina em vinho incluem, principalmente, a cromatografia $\operatorname{gasosa}^{5}$ (GC, "gas chromatography") e a cromatografia líquida de alta eficiência ${ }^{6-11}$ (HPLC, "high performance liquid chromatography"), sendo que as duas técnicas são seletivas e sensíveis, permitindo a detecção de pequenas quantidades de aminas. A análise de aminas bioativas em alimentos por HPLC tem aumentado, já que oferece a vantagem de separação e quantificação simultânea de aminas ${ }^{12}$. No entanto, a maioria das aminas não apresenta absorção ultravioleta

*e-mail: rzanella@base.ufsm.br

natural ou fluorescência. Por esta razão, a derivatização química é necessária para detectar as aminas por HPLC ${ }^{13}$.

Os agentes derivatizantes mais utilizados na determinação por fluorescência de aminas bioativas são: cloreto de dansyl, ortoftaldeído (OPA) e fluorescamine. A principal vantagem que o reagente OPA apresenta em relação aos outros reagentes derivatizantes é que este reage com aminas rapidamente e possibilita a detecção de baixos níveis de aminas biogênicas ${ }^{14}$. A Figura 1 representa a reação química entre a histamina e o reagente derivatizante OPA.

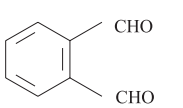

OPA

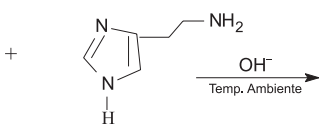

Histamina

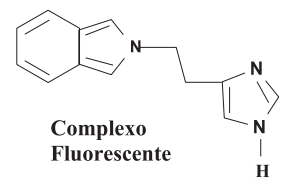

Figura 1. Reação de derivatização química da histamina com o agente derivatizante $O P A^{15}$

Uma vez desenvolvido um método de análise cromatográfica, é importante fazer a validação do mesmo para avaliar se fornece resultados confiáveis, de forma a poder ser aplicado rotineiramente. A validação consiste na avaliação da capacidade do processo analítico em produzir resultados compatíveis com precisão e exatidão consideradas satisfatórias. Um processo de validação bem definido e documentado oferece às agências reguladoras evidências objetivas de que os métodos e os sistemas são adequados para o uso desejado. Os parâmetros geralmente envolvidos no método de validação de métodos analíticos são curva analítica, linearidade, limite de detecção e limite de quantificação, exatidão, precisão e robustez ${ }^{16}$.

Este trabalho teve como objetivo desenvolver e validar um método analítico rápido e eficiente para determinação de histamina em vinhos empregando derivatização pré-coluna com OPA e quantificação por HPLC-fluorescência. O método desenvolvido foi aplicado em amostras de vinhos tintos obtidas por vinificações experimentais. 


\section{PARTE EXPERIMENTAL}

\section{Reagentes e soluções analíticas}

A água ultrapura foi obtida através do Sistema Milli-Q (Millipore, USA) e metanol grau HPLC adquirido da firma Mallinckrodt (USA). Foi preparada uma solução analítica estoque de $1000 \mathrm{mg} \mathrm{L}^{-1}$ de histamina (Sigma, Alemanha, pureza 98\%), através da dissolução do analito em solução aquosa de $\mathrm{HCl} 0,1 \mathrm{~mol}$ $\mathrm{L}^{-1}$. A partir desta solução, por diluições sucessivas, prepararam-se soluções analíticas nas concentrações de 10, 50, 100, 150 e $200 \mu \mathrm{g} \mathrm{L}{ }^{-1}$.

A solução derivatizante foi preparada da seguinte forma: $4,4 \mathrm{~g}$ de hidróxido de potássio p.a., 5,0 g de ácido bórico p.a., 0,04 g de ortoftaldeído (Acros, Bélgica, pureza 98\%) dissolvido em $300 \mu \mathrm{L}$ de metanol, $300 \mu \mathrm{L}$ de mercaptoetanol (Acros, Bélgica, pureza 99\%) e água Milli-Q suficiente para completar um volume de $100 \mathrm{~mL}$, obtendo-se uma solução com pH final 11,0. Este reagente é estável por 7 dias. Tanto as soluções analíticas como a solução derivatizante foram acondicionadas em frascos âmbar, mantidos sob refrigeração $\left(4^{\circ} \mathrm{C}\right)$.

A fase móvel foi preparada da seguinte forma: $2,72 \mathrm{~g}$ de acetato de sódio p.a., $180 \mu \mathrm{L}$ de trietilamina, $3,0 \mathrm{~mL}$ de tetraidrofurano (THF) e água Milli-Q suficiente para completar um volume de 400 $\mathrm{mL}$ foram misturados com $600 \mathrm{~mL}$ de metanol. $\mathrm{O} \mathrm{pH}$ foi ajustado para 7,2 com ácido acético glacial. A fase móvel foi desaerada por 10 min em banho de ultra-som à temperatura ambiente.

\section{Instrumentação}

Foi empregado um cromatógrafo a líquido equipado com bomba de HPLC Varian 9002 (USA); detector de fluorescência 821-FP, Jasco (Japão); coluna analítica Synergil C-18 (250 x 4,6 mm; $4 \mu \mathrm{m})$ (Phenomenex, USA) e um sistema de aquisição de dados Star Chromatography Workstation versão 4.5 (Varian, USA).

\section{Condições cromatográficas}

Foram adotados desde o início dos testes os comprimentos de onda de excitação em $340 \mathrm{~nm}$ e emissão em $445 \mathrm{~nm}^{17}$. A vazão de fase móvel utilizada foi de $1,2 \mathrm{~mL} \mathrm{~min}^{-1}$. Com esta vazão obteve-se uma boa separação e tempo total dos cromatogramas de $13 \mathrm{~min}$. O volume da alça de injeção foi de $20 \mu \mathrm{L}$.

\section{Preparo das amostras}

As amostras foram previamente centrifugadas a $4000 \mathrm{rpm}$ por 10 min em centrífuga para tubos Eppendorf de 1,5 mL. O sobrenadante foi transferido para outro tubo Eppendorf, que foi mantido em freezer até o momento das análises. Para as análises, as amostras foram retiradas do freezer e diluídas cem vezes (100 $\mu \mathrm{L}$ da amostra em balão volumétrico de $10 \mathrm{~mL}$ ) com a fase móvel.

\section{Procedimento de derivatização}

Para a obtenção da curva analítica, $500 \mu \mathrm{L}$ de cada solução analítica $\left(10,50,100,150\right.$ e $\left.200 \mu \mathrm{g} \mathrm{L}^{-1}\right)$ foram derivatizados com 500 $\mu \mathrm{L}$ de solução derivatizante em frascos de vidro com capacidade de $2 \mathrm{~mL}$. Após $2 \mathrm{~min}$ de reação, $20 \mu \mathrm{L}$ foram injetados no sistema, obtendo-se as áreas para a construção da curva analítica.

Para as amostras foram empregados $500 \mu \mathrm{L}$ da amostra diluída cem vezes para reagir com $500 \mu \mathrm{L}$ da solução derivatizante. Após exatamente 2 min de reação, a mistura foi filtrada em filtro de membrana $0,2 \mu \mathrm{m}$, tipo ponteira, diretamente no sistema de injeção do cromatógrafo.

\section{Avaliação da estabilidade do complexo histamina-OPA}

Foi realizada a avaliação da estabilidade do complexo histamina-OPA através de análises cromatográficas do padrão analítico de histamina submetido a diferentes tempos de reação com a solução derivatizante.

\section{Validação do método}

Definidas as melhores condições de separação da histamina, fase móvel, comprimento de onda e reação de derivatização, a etapa seguinte foi validar o método para análise de histamina, conforme os seguintes parâmetros:

\section{Linearidade}

No presente trabalho, considerou-se como faixa linear os pontos cujos valores de (S/Q)\% estão no intervalo de $100 \pm 5$, ou seja, pontos cujas razões sinal/concentração não diferem mais de $5 \%$ do coeficiente angular da reta (a). Para verificar se os pontos encontravam-se dentro da região linear da curva, foi utilizado o teste da razão entre o sinal (S) e a concentração $(\mathrm{Q})$, definida por:

$\left(\mathrm{S}_{\mathrm{i}} / \mathrm{Q}_{\mathrm{i}}\right) \%=\left(\mathrm{S}_{\mathrm{i}}-\mathrm{b} / \mathrm{Q}_{\mathrm{i}}\right) \mathrm{x} 100 / \mathrm{a}$

onde $\mathrm{b}$ é a inteseção da reta no eixo y quando $\mathrm{x}$ é igual a zero.

Os valores de $\mathrm{S}$ e $\mathrm{Q}$ foram obtidos na construção da curva analítica, através dos parâmetros de área do pico e concentração do analito $^{18}$.

\section{Limite de detecção (LOD) e limite de quantificação (LOQ)}

$\mathrm{Na}$ determinação do LOD do instrumento, foram feitas injeções em ordem decrescente de concentração até que o sinal cromatográfico atingisse uma área referente a três vezes o ruído da linha base, no tempo de retenção do pico de interesse. Para a determinação do LOQ, as injeções foram feitas até que a área do pico fosse dez vezes superior ao ruído da linha base no tempo de retenção do pico de interesse. Os LOD e LOQ do método foram obtidos multiplicando-se o valor de LOD e LOQ do instrumento por cem, valor referente à diluição da análise da amostra efetuada antes.

\section{Precisão}

$\mathrm{O}$ estudo da repetitividade do instrumento $\left(\mathrm{RSD}_{\mathrm{ri}}\right)$ foi realizado efetuando-se oito injeções da solução analítica correspondente ao ponto médio da curva analítica $\left(100 \mu \mathrm{g} \mathrm{L}^{-1}\right)$. A precisão intermediária do instrumento $\left(\mathrm{RSD}_{\text {pii }}\right.$ ) foi avaliada comparando-se os resultados obtidos por dois analistas em dias diferentes.

\section{Exatidão}

A recuperação, obtida a partir da fortificação, em 2 e $10 \mathrm{mg} \mathrm{L}^{-1}$, de uma amostra "branco", que apresentou valores de histamina $<$ LOD, foi utilizada para avaliar a exatidão do método. Em um balão volumétrico de $10 \mathrm{~mL}$, foram adicionados $100 \mu \mathrm{L}$ da amostra "branco", $1 \mathrm{~mL}$ da solução padrão e o volume foi completado com fase móvel. Este ensaio foi realizado em triplicata. Paralelamente a isto, soluções analíticas contendo 0,2 e $1,0 \mathrm{mg} \mathrm{L}^{-1}$ foram diluídas cem vezes, derivatizadas e injetadas, em triplicata, no sistema cromatográfico. As respectivas médias das concentrações obtidas representaram o valor referencial, ou seja, o valor de concentração que deveria ser teoricamente encontrado. 


\section{Robustez}

Segundo Ribani et $a l .{ }^{16}$ a robustez de um método mede a sensibilidade que este apresenta face a pequenas variações. Um método é considerado robusto quando não é afetado por uma modificação pequena e deliberada em seus parâmetros.

A robustez do método proposto foi avaliada mediante a utilização de fases móveis variando o $\mathrm{pH}$ final em $\pm 0,2$ unidades. Também se avaliou a influência de pequenas variações do tempo de derivatização (1,8; 2,0 e 2,2 min).

\section{RESULTADOS E DISCUSSÃO}

A Figura 2 representa o cromatograma obtido na análise da solução analítica contendo $100 \mu \mathrm{g} \mathrm{\textrm {L } ^ { - 1 }}$ de histamina (A) e de uma das amostras avaliadas neste trabalho (B). Com a análise das amostras de vinho utilizadas neste trabalho foi verificado que o método não apresentou interferências no tempo de retenção do composto em estudo.

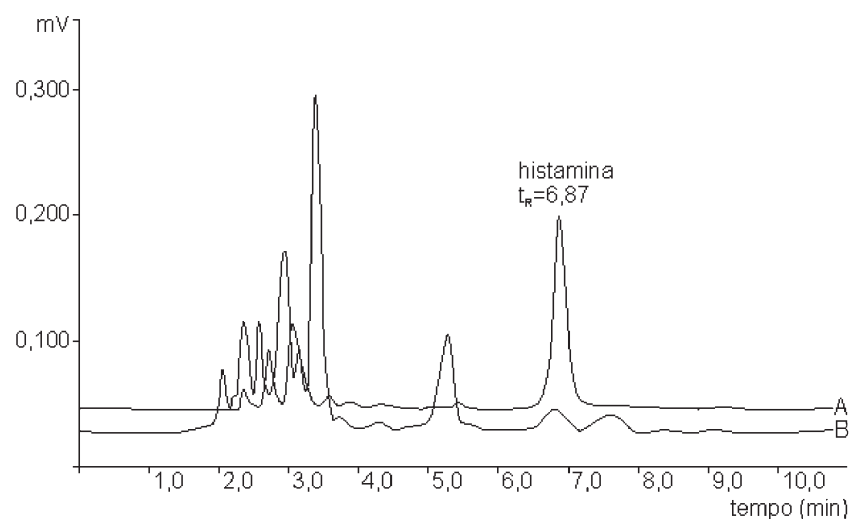

Figura 2. Cromatograma típico de solução analítica contendo $100 \mu \mathrm{g} L^{-1}$ de histamina (A) e de uma amostra (B)

A Tabela 1 contém os resultados obtidos no cálculo de linearidade para os pontos da curva analítica, a equação da curva utilizada para quantificação da histamina e o coeficiente de determinação.

Tabela 1. Resultados referentes ao estudo da linearidade tendo como base a concentração de histamina e a área média obtida após as injeções no sistema cromatográfico

\begin{tabular}{lcc}
\hline Concentração $\left(\mu \mathrm{g} \mathrm{L}^{-1}\right)$ & Área média & S/Q $(\%)$ \\
\hline 10 & 12560 & 104,8 \\
50 & 60656 & 103,1 \\
100 & 112804 & 96,1 \\
150 & 177240 & 100,8 \\
200 & 235151 & 100,3 \\
\hline
\end{tabular}

Equação da curva analítica para histamina: $y=1170,6 x+282,4$; Coeficiente de determinação $r^{2}=0,9992$

A partir dos valores obtidos, conclui-se que o modelo é adequado, pois o coeficiente de determinação $\left(r^{2}\right)$ foi maior que 0,999 , considerado como evidência de um ajuste ideal dos dados para a linha de regressão ${ }^{19}$.

De acordo com dados da literatura, Mafra et $a l^{2}{ }^{2}$ desenvolveram um método cromatográfico para quantificar aminas bioativas em vinhos portugueses no qual foi obtido um intervalo linear para quantificação de histamina de 0,091 a $1,822 \mathrm{mg} \mathrm{L}^{-1}$. Em outro trabalho, Crespo e Lasa ${ }^{20}$ encontraram como intervalo confiável para quantificação de histamina valores que variaram de 0,55 a $15,55 \mathrm{mg} \mathrm{L}^{-1}$.
Avaliando-se os resultados obtidos, conclui-se que a curva analítica apresentou linearidade satisfatória na faixa entre 0,01 e $0,2 \mathrm{mg} \mathrm{L}^{-1}$, uma vez que as razões S/Q para os pontos da curva analítica não se desviaram mais que $5 \%$ em relação ao coeficiente angular da reta.

Os valores de $\mathrm{LOD}_{\mathrm{i}}$ e $\mathrm{LOQ}_{\mathrm{i}}$ do instrumento foram $0,0025 \mathrm{e}$ $0,005 \mu \mathrm{g} \mathrm{L}^{-1}$, respectivamente. Os $\mathrm{LOD}_{\mathrm{m}} \mathrm{e} \mathrm{LOQ}_{\mathrm{m}}$ do método foram 0,25 e $0,5 \mathrm{mg} \mathrm{L}^{-1}$, ou seja, cem vezes os valores dos limites para o instrumento, uma vez que as amostras foram diluídas cem vezes para análise.

Romero et $a l .{ }^{21}$ descreveram um método para quantificação de oito aminas bioativas em vinhos com LOD do método para histamina de $0,167 \mathrm{mg} \mathrm{L}^{-1}$. Em trabalho desenvolvido por Mafra et al. ${ }^{2}$, foi encontrado um LOD do método de $0,93 \mathrm{mg} \mathrm{L}^{-1}$ para a quantificação de histamina em vinhos portugueses. Portanto, o método desenvolvido em nosso trabalho apresentou sensibilidade semelhante, com $\mathrm{LOD}_{\mathrm{m}}$ de $0,25 \mathrm{mg} \mathrm{L}^{-1}$.

A Tabela 2 apresenta a repetitividade e precisão intermediária do instrumento, em termos de área da solução analítica referente ao ponto médio da curva analítica, e do método obtido para uma amostra fortificada.

Tabela 2. Resultados de precisão em termos de $\mathrm{RSD}_{\mathrm{ri}}$ e $\mathrm{RSD}_{\text {pii }}$ do instrumento obtido a partir da solução analítica $100 \mu \mathrm{g} \mathrm{L}^{-1}$, e de $\mathrm{RSD}_{\mathrm{rm}}$ e $\mathrm{RSD}_{\text {pim }}$ do método obtido a partir de amostra fortificada em $2 \mathrm{mg} \mathrm{L}^{-1}$

\begin{tabular}{llll}
\hline \multicolumn{2}{r}{ Repetitividade (\%) } & \multicolumn{2}{c}{ Precisão intermediária (\%) } \\
\hline RSD $_{\text {ri }}$ & $\mathrm{RSD}_{\mathrm{rm}}$ & $\mathrm{RSD}_{\text {pii }}$ & $\mathrm{RSD}_{\text {pim }}$ \\
3,7 & 6,0 & 5,6 & 2,9 \\
\hline
\end{tabular}

$\mathrm{n}=8$ repetições para cada ensaio de precisão

Os resultados, em termos de repetitividade e precisão intermediária obtidos para o instrumento são aceitáveis, uma vez que estão dentro do limite aceito para validação de métodos cromatográficos que é de $5 \%$. Outros trabalhos apresentaram valores de desvio padrão relativo (RSD) de $1,77^{20}, 1,21^{11}$ e $7,3 \%{ }^{23}$, sendo este último valor acima do aceitável. Os resultados de precisão obtidos para o método mostraram-se aceitáveis, estando dentro do limite aceito para validação de métodos cromatográficos que deve ser menor que $15 \%$.

A Tabela 3 fornece os valores de recuperação (\%) obtidos através da aplicação do método desenvolvido em uma amostra "branco" fortificada em 2 e $10 \mathrm{mg} \mathrm{L}^{-1}$. Os resultados de recuperação para este trabalho são satisfatórios para as concentrações estudadas pois estão dentro dos valores aceitos, os quais devem estar entre 70 e $120 \%$. Em trabalho realizado com vinhos portugueses ${ }^{2}$ foi obtida uma recuperação de $74,5 \%$, abaixo da recuperação apresentada pelo método proposto.

Tabela 3. Valores de recuperação encontrados após fortificação de amostra "branco" em dois níveis de fortificação

\begin{tabular}{ccc}
\hline Nível de fortificação $\left(\mathrm{mg} \mathrm{L}^{-1}\right)$ & Recuperação $(\%)$ & Média (\%) \\
\hline \multirow{2}{*}{2} & 93,7 & \\
& 90,7 & 95,5 \\
& 102,2 & \\
\multirow{2}{*}{10} & 84,6 & \\
& 95,8 & 89,9 \\
\hline
\end{tabular}

\section{Avaliação da estabilidade do complexo histamina-OPA}

A Figura 3 apresenta os cromatogramas que foram obtidos para uma solução de histamina na concentração de $500 \mu \mathrm{g} \mathrm{L}^{-1}$, submeti- 


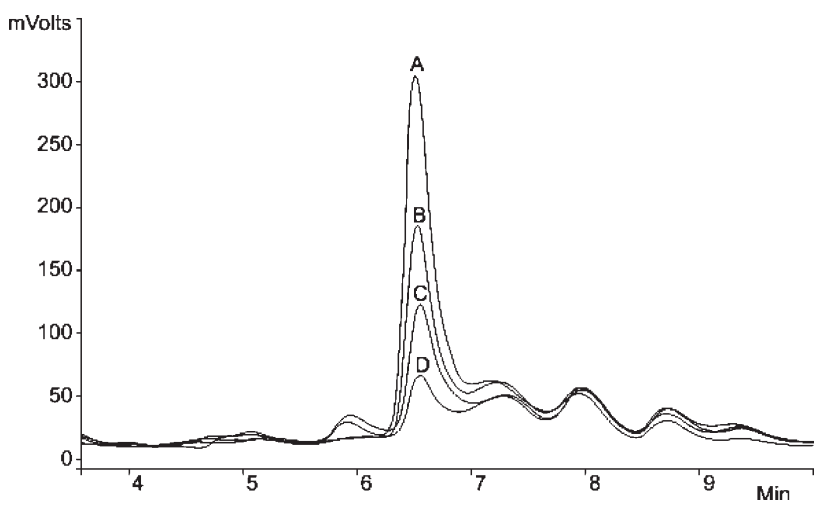

Figura 3. Cromatogramas obtidos na avaliação da estabilidade da solução analítica $500 \mu \mathrm{g} \mathrm{L}^{-1}$ de histamina derivatizada com ortoftaldeído, analisados após diferentes tempos do início da reação, em min: $A=1 ; B=10 ; C=15$ e $D=20$

da a diferentes tempos de reação com o derivatizante ortoftaldeído.

A reação de derivatização é quase instantânea e observou-se que o complexo histamina-OPA é estável até 4 min após o início da reação, apresentando áreas semelhantes. Entretanto, após $10 \mathrm{~min}$ de reação observou-se uma diminuição significativa do sinal, indicando uma degradação pronunciada do complexo fluorescente. Este fato foi também relatado por outros autore ${ }^{23,24}$. Trabalho realizado por Douabalé et al. ${ }^{15}$ mostrou que o complexo fluorescente formado é inibido em meio ácido, e em meio alcalino um aumento da fluorescência foi observado, mas o complexo histamina-OPA foi instável. Apesar disso, um estudo cinético mostrou que uma boa correlação linear entre a máxima fluorescência do complexo formado e a concentração de histamina poderia ser obtida em meio alcalino, como foi empregado no método proposto. Assim, para a determinação de histamina, adotou-se exatamente 2 min como tempo de reação, injetando-se imediatamente após este tempo.

As pequenas variações do $\mathrm{pH}$ da fase móvel e do tempo de derivatização, testadas na etapa de validação, não influenciaram na resposta do método, indicando que o mesmo é robusto.

\section{Aplicação do método proposto}

Foram analisadas 21 amostras de vinhos tintos obtidos de vinificações experimentais, realizadas com o objetivo de monitorar os níveis de histamina durante as fermentações alcoólica e malolática. A histamina foi encontrada em todas as amostras, com concentrações que variaram de 1,2 a $5,7 \mathrm{mg} \mathrm{L}^{-1}$, apresentando valor médio de $3,7 \mathrm{mg} \mathrm{L}^{-1}$.

\section{CONCLUSÃO}

O método proposto apresentou ótimos resultados em todas as etapas de validação, mostrando ser sensível, preciso e exato. A etapa de derivatização de fácil execução, aliada com a detecção por fluorescência, dispensa uma extensa preparação da amostra, bem como uma etapa de "clean up" para eliminação de interferentes. A avaliação da estabilidade do complexo indicou a necessidade de efetuar a derivatização somente instantes antes da análise cromatográfica. Em comparação com outros métodos, este apresentou melhores resultados nos parâmetros avaliados, mostrando-se adequado para a quantificação de histamina em amostras de vinho tinto. O método apresentou resposta linear para histamina nas amostras até pelo menos $20 \mathrm{mg} \mathrm{L}^{-1}$. Nas 21 amostras de vinho analisadas, a concentração de histamina variou entre 1,2 e $5,7 \mathrm{mg} \mathrm{L}^{-1}$.

\section{AGRADECIMENTOS}

Ao CNPq e à CAPES pelo apoio financeiro e bolsas.

\section{REFERÊNCIAS}

1. Coton, E.; Rollan, G.; Bertrand, A.; Lonvaud-Funel, A.; Am. J. Enol. Vitic. 1998, 49, 199.

2. Mafra, I.; Herbert, P.; Santos, L.; Barros, P.; Alves, A.; Am. J. Enol. Vitic. 1999, 50, 128 .

3. Bataglia, R.; Frölich, D.; Commun. 1978, 2, 100.

4. Herbert, P.; Santos, L.; Alves, A.; J. Food Sci. 2001, 66, 1319

5. Fernandes, J. O.; Ferreira, M. A.; Chromatographia 2000, 52, 77.

6. Beljaars, P. R.; van Dijk, R.; Jonker, K. M.; Schout, L. J.; J. AOAC Int. 1998, 81, 991.

7. Garcia-Villar, N.; Saurina, J.; Hernández-Cassou, S.; Analyst 2005, 130, 1286.

8. Gennaro, M. C.; Abrigo, C.; Chromatographia 1991, 31, 381.

9. Loukou, Z.; Zotou, A.; Chromatographia 2003, 58, 579.

10. Subden, R. E.; Duitschaever, C.; Kaiser, K. ; Noble, A. C.; Am. J. Enol. Vitic. 1979, 30, 19.

11. Vidal-Carou, M. C.; Lahoz-Portolés, F.; Bover-Cid, S.; Mariné-Font, A.; J. Chromatogr., A 2003, 998, 235.

12. Bauza, T.; Blaise, A.; Daumas, F.; Cabanis, J. C.; J. Chromatogr., A 1995, 707, 373.

13. Glória, M. B. A.; Daeschel, M. A.; Watson, B.; Simon-Sarkadi, L.; Am. J. Enol. Vitic. 1998, 49, 280.

14. Busto, O.; Miracle, M.; Guasch, J.; Borrull, F.; J. Chromatogr., A 1997, 757, 311.

15. Douabalé, S. E.; Mactar, D.; Atanasse, C.; Alphonse, T.; Talanta 2003, 60, 581.

16. Ribani, M.; Bottoli, C. B. G.; Collins, C. H.; Jardim, I. C. S. F., Melo, L. F. C.; Quim. Nova 2004, 27, 771.

17. Vale, S. R.; Glória, M. B. A.; J. AOAC. Int. 1997, 80, 1006.

18. Chasin, A. A. M.; Nascimento, E. S.; Ribeiro Neto, L. M.; Siqueira, M. E. P. B.; Andraus, M. H.; Salvatori, M. C.; Fernícola, N. A. G.; Gorni, R.; Salcedo, S.; Rev. Brasil. Toxicol. 1998, 11, 1.

19. Shabir, G. A.; J. Chromatogr., A 2003, 987, 57.

20. Crespo, M. I.; Lasa, B. V.; Am. J. Enol. Vitic. 1994, 45, 460.

21. Romero, R.; Gázquez, D.; Bagur, M. G.; Sánchez-Viñas, M.; J. Chromatogr., A 2000, 871, 75 .

22. Causon, R.; J. Chromatogr., B: Anal. Technol. Biomed. Life Sci. 1998, 689, 175.

23. Male, K. B.; Luong, J. H. T.; J. Chromatogr., A 2001, 926, 309.

24. Buteau, C.; Duitschaever, C. L; Ashton, G. C.; J. Chromatogr. 1984, 284, 201. 